\title{
Measurement of dielectric loss tangent at cryogenic temperature using superconducting film resonator
}

\author{
Yufang Zhang $^{1} \cdot$ Zhenqing Wang $^{1}$
}

Received: 4 October 2015/ Accepted: 19 October 2015/Published online: 5 November 2015

(C) The Author(s) 2015. This article is published with open access at Springerlink.com

\begin{abstract}
We demonstrate that the superconducting film resonator can be used to accurately and quantitatively measure the microwave dielectric loss tangent of a variety of materials. Compared to traditional dielectric resonator loaded metal cavity method, it has advantage of small sample size $(\sim 2-3$ orders of magnitude smaller than the old method), and much higher sensitivity to measure small loss tangent values as small as $10^{-5}$ at around $7 \mathrm{GHz}$ band at cryogenic temperatures. This method can be utilized widely in study of mechanism of microwave loss at cryogenic temperature range, which is extremely important in superconducting microwave application areas, such as novel super quantum computers.
\end{abstract}

Keywords Dielectric loss tangent $\cdot$ Superconducting plate resonator $\cdot$ Cryogenic temperature

\section{Introduction}

Ultrahigh performance microwave dielectrics have been widely used in microwave passive devices including resonators and filters in the area of telecommunication systems and photonics [1-9]. Low microwave loss tangent, high dielectric constant and temperature stability are desired to achieve high device performance. In these three parameters, the mechanism of dielectric loss tangent is the one that has been studied by many researchers but still not understood completely [1-3]. Recently, abnormal behavior

Yufang Zhang

yufang.zhang1@yahoo.com

1 College of Physics and Electronic Engineering, Northwest Normal University, Lanzhou 730070, Gansu, China of microwave loss tangent of dielectric materials at cryogenic temperature range, including high-k, low-loss commercial $\mathrm{Ba}\left(\mathrm{Zn}_{1 / 3} \mathrm{Ta}_{2 / 3}\right) \mathrm{O}_{3}$, $(\mathrm{Zr}, \mathrm{Sn}) \mathrm{TiO}_{4}, \mathrm{Ba}\left(\mathrm{Zn}_{1 / 3} \mathrm{Nb}_{2 / 3}\right) \mathrm{O}_{3}$ with different dopants, has been studied [10-14]. Absorption of microwave energy by unpaired spins in $d$-shell electrons in transition-metal dopant ions has been attributed to the root cause for the high microwave loss of a series of microwave ceramic materials at cryogenic temperatures. Traditionally, the microwave loss tangent of ceramic materials is measured using the $\mathrm{TE}_{01 \delta}$ mode of a ceramic resonator mounted in a metal cavity $[1,2]$. In this method, usually a ceramic cylinder sample with diameter of a few centimeters is loaded in a metal cavity with size of about three times larger than the ceramic sample. The microwave loss due to conductivity of metal cavity wall often limits the sensitivity of loss tangent measurement. Superconducting parallel plate resonator (PPR), a parallel plate capacitor structure with two superconducting films back to back and a dielectric spacer between them was first introduced by Taber [15] to measure the RF surface resistance of superconductor films. This method was then extensively utilized in research studies [16-22]. In this manuscript, we, in another point of view, will demonstrate that this device can also be used to measure microwave loss tangent of any dielectric materials at cryogenic temperature range with great advantages over the commonly used $\mathrm{TE}_{01 \delta}$ metal cavity method $[1,2]$. Besides the high sensitivity of loss tangent measurement, the sample size needed for PPR is almost 2-3 orders smaller than that needed for cavity method. Also, since a single small high-loss defective region of a bulk sample can skew the overall quality factor performance, this method can be used to determine the material uniformity by measuring many samples from a single ceramic bulk sample. In this paper, we first explain the experimental setup and measurement capability of this 
technique, and then use Ni-doped BZT as a case study example to show its power to characterize microwave loss of dielectric materials at cryogenic temperature.

\section{Theoretical principle and experiment method}

The PPR consists of two superconducting films and one dielectric spacer, and the spacer is placed between these two superconductor films to form a sandwich structure. The resonator is then placed inside a small cavity made of oxygen free high-conductivity copper (OFHC) which is gold (purity $>99.9 \%$ ) plated to minimize the radiation loss. The details of this device are well described in some references [15-20]. Here, we use sputtered $\mathrm{Nb}$ films (for measurement up to $9 \mathrm{~K}$ ), and $\mathrm{YBa}_{2} \mathrm{Cu}_{3} \mathrm{O}_{7-\delta}(\mathrm{YBCO})$ films deposited on sapphire substrates (for measurement up to $80 \mathrm{~K})$ by sputtering deposition. For the dielectric spacer, we choose several different materials. These include 2-in. silicon wafers (Virginia Semiconductor Inc., Fredericksburg, VA, USA) of both undoped $\left[25 \mu \mathrm{m}\right.$ thick ultrathin ${ }^{\circledR}$, (100) orientation, resistivity $>20 \Omega-\mathrm{cm}]$ and lightly doped n-type $\left(125 \mu \mathrm{m}\right.$ thick) ones, Teflon ${ }^{\circledR}$ FEP films (Du Pont Electronics, Wilmington, DE, USA, $12.5 \mu \mathrm{m}$ thick), twoside polished $c$-sapphire substrates $(1 \mathrm{~cm} \times 1 \mathrm{~cm} \times$ $0.5 \mathrm{~mm})$, and thin chips $\mathrm{Ba}\left(\mathrm{Zn}_{1 / 3} \mathrm{Ta}_{2 / 3}\right) \mathrm{O}_{3}$ ceramics doped with different amount of $\mathrm{Ni}$ ion substituting $\mathrm{Zn}$ position [one can think it as a solid alloy of $x \mathrm{Ba}\left(\mathrm{Ni}_{1 / 3} \mathrm{Ta}_{2 / 3}\right) \mathrm{O}_{3}$ $(1-x) \mathrm{Ba}\left(\mathrm{Zn}_{1 / 3} \mathrm{Ta}_{2 / 3}\right) \mathrm{O}_{3}$ ] [10-14]. The ceramic was sintered by traditional powder processing method [1-3]. The Ni-doped BZT sample was processed as described in [1014]. First, the pure $\mathrm{BZT}$ and $\mathrm{Ba}\left(\mathrm{Ni}_{1 / 3} \mathrm{Ta}_{2 / 3}\right) \mathrm{O}_{3}$ (BNT) powder were synthesized separately. Then, the BZT and BNT powder were mixed into slurry according to desired atomic ratio by ball milling. The resulting mixed powder was then used to sinter Ni-doped BZT ceramic samples. The undoped and lightly doped silicon wafers in this work will come into carrier freeze-out zone at $4.2 \mathrm{~K}$ and shows a very low microwave loss tangent. The cavity loaded with the sample is then affixed to the end of a cryogenic dipping probe which is then inserted into the liquid Helium Dewar. Two $50-\Omega$ semi-rigid coaxial microwave cable was put inside the dipping probe, at one ends with metal outer conductor peeled off to make coupling microwave feeding probes for the ceramic sample in the metal cavity, and at the other end connected to the HP 8510C vector network analyzer, from which $S_{12}$ or $S_{21}$ network parameters can be collected. The coupling probes can be moved close or away from the resonator to excite resonance modes. These values are fit to a circle in the Smith chart to infer the quality factor. The distance between coupling loops and sample is adjusted to be weakly coupled so that the unloaded $Q$ can be determined to better than a few percent. Because only the value of the quality factor is sufficient to infer the loss tangent, a calibrated measurement is not required. The input source power should be chosen neither too large in case of the nonlinear effect $[16,20]$, nor too small to get a good signal to noise ratio of the $S_{12}$ or $S_{21}$ signal intensity. It should be kept above $10-15 \mathrm{db}$ to measure an accurate $3-\mathrm{db}$ bandwidth of the transmission coefficient. The distance between tips of coaxial probes and the resonator was adjusted to be within tenths of a millimeter to get a weak coupling, in which case the loaded $Q$ and unloaded $Q$ is approximately equal to each other. Usually, at the beginning of the experiment, the probes were moved barely touch the sample. During the experiment, the distance was increased to lower the coupling coefficient until the weak coupling needed reached. The sharp peaks in $S_{21}$ or $S_{12}$ diagram correspond to the resonant modes. These modes can be distinguished from the cavity modes easily by observing the shift of resonate frequency [15-17] with the change of temperature, while the cavity modes do not shift obviously.

The overall unloaded quality factor $Q$ of the resonator can be well explained by the equation [15]

$Q^{-1}=Q_{\mathrm{d}}^{-1}+Q_{\mathrm{r}}^{-1}+Q_{\mathrm{c}}^{-1}=\tan \delta+\alpha_{\mathrm{s}}+\left(\beta R_{\mathrm{s}} / s\right)$

where $\tan \delta$ is the loss tangent of dielectric spacer. The second term is radiation loss which is due to energy radiated from the surrounding of the resonator, and the third term is conduction loss caused by surface resistance of superconductor films. Our goal is to measure $\tan \delta$ of dielectric materials, if in some situation that $\tan \delta$ can dominate over the other two terms in Eq. 1, one can directly measure $Q$ and use $Q^{-1}$ to replace $\tan \delta$ without caring about the conduction loss and radiation loss terms. The radiation loss is proportional to the spacer thickness. $\alpha$ is a parameter that gives information about the radiation loss. It changes with the dielectric constant of the spacer. Usually, high $\varepsilon_{\mathrm{r}}$ spacers, e.g., $\mathrm{LaAlO}_{3}\left(\varepsilon_{\mathrm{r}}=25\right)$ and $\mathrm{Ba}\left(\mathrm{Zn}_{1 / 3} \mathrm{Ta}_{2 / 3}\right) \mathrm{O}_{3}\left(\varepsilon_{\mathrm{r}}=29\right)$ instead of Teflon $\left(\varepsilon_{\mathrm{r}}=2.1\right)$, can reduce radiation loss of the PPR and lead to higher unloaded $Q$. An inverse square root law between radiation loss and dielectric constant can be derived [15], so the higher dielectric constant, the lower radiation loss, i.e., lower $\alpha$. The conduction loss is inversely proportional to thickness of spacers. $\beta$ is given by $\beta=1 /\left(f \times \pi \mu_{0}\right)=3.9 \times 10^{-5} \mathrm{~m} \Omega^{-1}$ at $6.5 \mathrm{GHz}$, where $\mathrm{f}$ is resonant frequency [15].

\section{Results and discussion}

The summary of PPR measurement with a series of spacer materials and $\mathrm{Nb}$ films at $4.2 \mathrm{~K}$ is listed in Table 1 . Note that an unloaded $Q$ as high as 160,000 was obtained at 
Table 1 Summary of the PPR measurement with different spacer materials at the temperature of $4.2 \mathrm{~K}$ using $\mathrm{Nb}$ films

\begin{tabular}{lccccccr}
\hline Spacer material & \multicolumn{1}{c}{$\varepsilon_{\mathrm{r}}$} & $t(\mu \mathrm{m})$ & \multicolumn{1}{c}{$Q_{\mathrm{u}}$} & $f_{0}(\mathrm{GHz})$ & \multicolumn{1}{c}{$Q_{\mathrm{u}}^{\mathrm{a}}$} & \multicolumn{1}{c}{$Q_{\mathrm{d}}^{\mathrm{a}}$} & \multicolumn{1}{c}{$\tan \delta^{\mathrm{a}}$} \\
\hline Silicon & 11.7 & 175 & 158,590 & 6.8 & 165,910 & 840,336 & $1.19 \times 10^{-6}$ \\
C-sapphire & 11.5 & 500 & 59,123 & 6.85 & 62,307 & 121,951 & $8.2 \times 10^{-6}$ \\
$\mathrm{Ba}\left(\mathrm{Zn}_{1 / 3} \mathrm{Ta}_{2 / 3}\right) \mathrm{O}_{3}$ & 29.0 & 500 & 66,801 & 6.91 & 71,015 & 116,279 & $8.6 \times 10^{-6}$ \\
Teflon FEP & 2.1 & 12.5 & 17,696 & 11.08 & 30,165 & 273,224 & $3.66 \times 10^{-6}$ \\
\hline
\end{tabular}

${ }^{\text {a }}$ Means the values were scaled to $6.5 \mathrm{GHz}$
6.8 GHz for Silicon spacer with thickness of $175 \mu \mathrm{m}$. This sets a record for the highest $Q$ factor we measured, ten times higher compared to $\sim 15,000$ at the same frequency range by metal cavity method using high- $k$, low-loss dielectric material resonator, like BZT. This is due to the super-low surface resistance of $\mathrm{Nb}$ superconducting films and very low dielectric loss of silicon at $4.2 \mathrm{~K}$. Other materials, such as single crystal sapphire substrate, BZT ceramic chip has smaller $Q$ factor due to higher material microwave loss. $12.5 \mu \mathrm{m}$ thick Teflon film was routinely used to determine the surface resistance of superconductor films. The resonant frequency is around $11 \mathrm{GHz}$ with $Q$ of 18,000 , and the calculated surface resistance $R_{\mathrm{S}}$ is around $10 \mu \Omega$ scaled to $6.5 \mathrm{GHz}$ at $4.2 \mathrm{~K}$. The surface resistance of the superconductor films will increase as the temperature goes up according to BCS theory. When it is close to $T_{\mathrm{c}}, R_{\mathrm{s}}$ increases dramatically due to loss of superconductivity and the PPR resonant modes will disappear in the network analyzer. The temperature dependence of $R_{\mathrm{S}}$ of the $\mathrm{Nb}$ films in this study is summarized in Fig. 1. As is shown, when temperature changes from 4.2 to $6 \mathrm{~K}, R_{\mathrm{s}}$ increases by about four times, and the sensitivity of measuring loss tangent of the dielectric spacer is highly reduced.

As mentioned in the introduction section, our goal is to measure $\tan \delta$ of dielectric materials, we prefer $\tan \delta$ to dominate over the other two terms in Eq. 1, so that one can directly measure $Q$ and use $Q^{-1}$ to replace $\tan \delta$ without

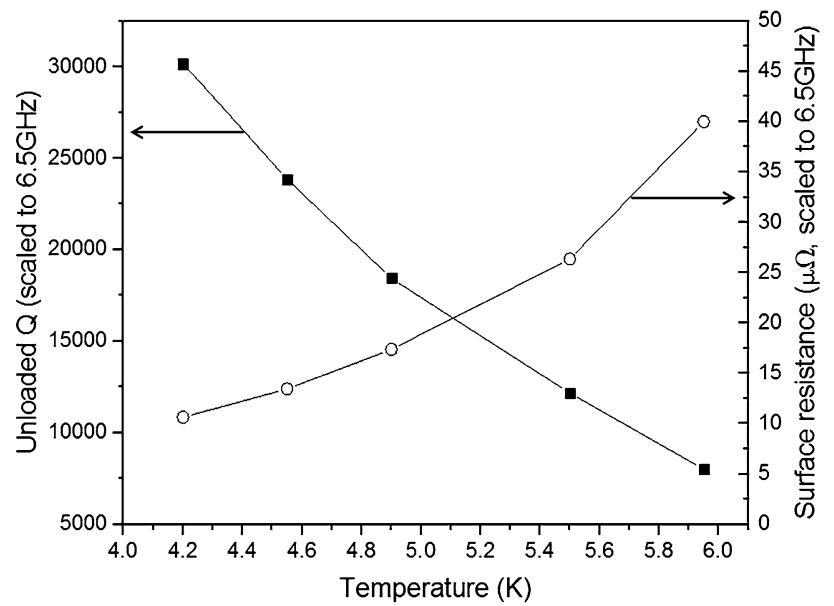

Fig. 1 Unloaded $Q$ and corresponding surface resistance of superconducting $\mathrm{Nb}$ films as a function of temperature caring about the conduction loss and radiation loss terms. As we take a deeper investigation of Eq. 1, we find that the radiation loss is scaling with $s$ ( $s$ is thickness of spacer), conduction loss is proportional to $1 / s$, and $\tan \delta$ is a constant independent of s. Thus, there must be a special value of $s$, for which the summation of radiation loss and conduction loss will be minimal and $Q$ will achieve its peak value. Figures 2 and 3 summarize the relation between the spacer thickness and unloaded $Q$ factor of PPR, as well as equivalent surface resistance $\hat{R}_{\mathrm{s}}$. As shown in Fig. 2, the optimal thickness $t_{\text {MAX }}$ for measuring loss tangent is around $175 \mu \mathrm{m}$ for $\mathrm{Si}$ spacers. At small spacer thickness $(<100 \mu \mathrm{m})$, the unloaded $Q$ increases almost linearly with it, because the surface resistance dominates in this range. On the other side, at larger thickness, the radiation loss begins to dominate. At the intermediate range, around $175 \mu \mathrm{m}$, the unloaded $Q$ reaches its maximum, about 165,000 at $6.5 \mathrm{GHz}$. Also, at $500 \mu \mathrm{m}$, the unloaded $Q$ is still better than 120,000 . Silicon wafers were chosen as spacers to study the $Q$ versus thickness relation because ultra-thin undoped wafers ( $25 \mu \mathrm{m}$ thick) are available with

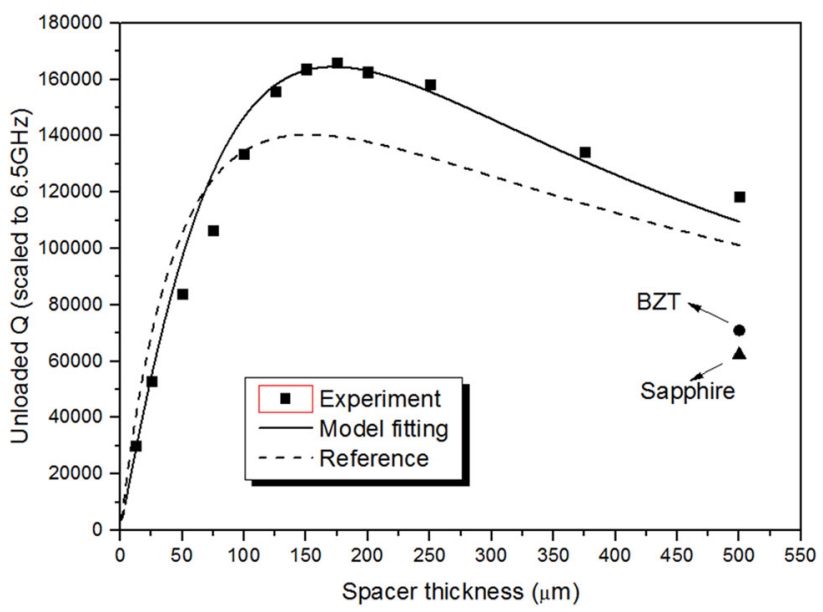

Fig. 2 Unloaded $Q$ versus spacer thickness of parallel plate resonator (PPR) using low-loss spacers. The first dot of the thickness $12.5 \mu \mathrm{m}$ was measured using the Teflon film, those between 25 and $100 \mu \mathrm{m}$ were measured using the $25 \mu \mathrm{m}$ thick undoped silicon wafer, and those between 125 and $500 \mu \mathrm{m}$ were measured using the $125 \mu \mathrm{m}$ thick lightly doped n-type silicon wafers. The dots are experimental data, the solid line is model curving fitting and the dashed line is from the data in Ref. [15] 
smooth surfaces. They can be easily stacked together to obtain different thickness between 25 and $500 \mu \mathrm{m}$. Clamping them tightly can minimize the influence of the air gap between layers. On the other hand, Sapphire substrates usually have low loss, but they are usually thick $(500 \mu \mathrm{m})$ and has high hardness so it is very difficult to polish them into different thickness. $\mathrm{Ba}\left(\mathrm{Zn}_{1 / 3} \mathrm{Ta}_{2 / 3}\right) \mathrm{O}_{3}$ ceramics could be another candidate for polishing because of its relatively low hardness, but it is very brittle and easy to break during the machining. The equivalent surface resistance $\hat{R}_{\mathrm{S}}$ [15] is defined by

$\hat{R}_{\mathrm{S}}=s /(Q \beta)=R_{\mathrm{S}}+s^{2} \alpha / \beta+s \tan \delta$.

It is a quadratic polynomial equation of thickness s. We can fit this formula to the experimental data shown in Fig. 3, and extract the surface resistance $R_{\mathrm{S}}$ of films, the $\tan \delta$ of the dielectric spacer and the radiation parameter $\alpha$. In this work, at $6.5 \mathrm{GHz}, R_{\mathrm{S}}$ was determined to be $10.8 \mu \Omega$ for the $\mathrm{Nb}$ film, $\tan \delta$ was $1.19 \times 10^{-6}$ for silicon spacers and $\alpha$ was $1.42 \times 10^{-2} \mathrm{~m}^{-1}$ for the PPR with Silicon spacers. The parameters in Taber's work were scaled to $12 \mathrm{GHz}$ [15]. Using the scaling rule of $R_{\mathrm{S}}$ with frequency, $R_{\mathrm{S}} \sim \omega^{2}$, the parameters can be scaled to $6.5 \mathrm{GHz}$, which gives $R_{\mathrm{s}}$ of $6.75 \mu \Omega, \tan \delta$ of $3.66 \times 10^{-6}$ for Teflon films and $\alpha$ of $1.14 \times 10^{-2} \mathrm{~m}^{-1}$. Compared with $\mathrm{Nb}$ films used in our work, their films have lower surface resistance, but the dielectric spacer we used, Silicon, is better than Teflon. The results with other dielectric spacers are listed in Table 1. Their dielectric constant ranges from about 2 to 30 . The quality factor of the spacer material $Q_{\mathrm{d}}$ and $\tan \delta$ is calculated and summarized in Table 1 using these parameters obtained by curve fitting and measured $Q$ factor data. Normally, sapphire is considered as very low-loss material, and other researchers claimed measured loss of $\sim 10^{-8}$ at

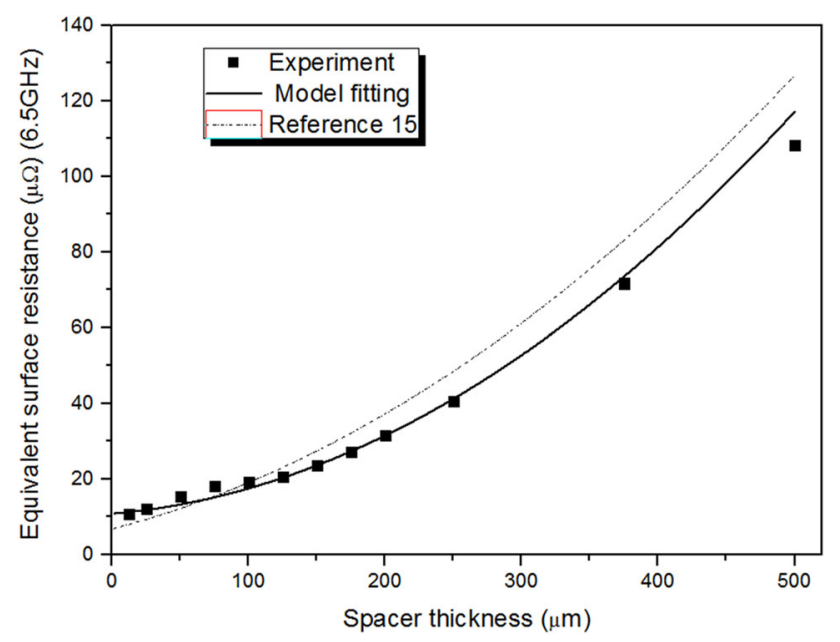

Fig. 3 Equivalent surface resistance of the PPR using low-loss spacers liquid helium temperature. But our measured loss is not so low, because there might be trace amount of transitionmetal ions impurities in the sample which cause paramagnetic loss. For $175 \mu \mathrm{m}$ thick silicon spacer, the summation of conduction loss and radiation loss is $5 \times 10^{-6}$ at 6.5 GHz. This implies that one can measure a dielectric material loss of $5 \times 10^{-5}$ at $6.5 \mathrm{GHz}$ with error within $10 \%$.

From explanation in the last paragraph, there is an optimal spacer thickness range around $175 \pm 50 \mu \mathrm{m}$, where the loss tangent measurement has the best sensitivity. Also, the thinner the spacer material sample, the more difficult it can be prepared (dicing and polishing) and handled. Most of the time, during the preparation of ceramic chip sample $\sim 175 \mu \mathrm{m}$ thick, it ends up with cracking. So, a thicker spacer material is desired without reduction in the measurement sensitivity. Since the radiation loss coefficient $\alpha \sim 1 / \sqrt{\varepsilon_{\mathrm{r}}}$, one can imagine that using materials with different dielectric constant, the optimal spacer thickness $t_{\text {MAX }}$ should be different. We can see this from Fig. 4. It shows the calculated $Q$ versus spacer thickness relationship only changing the different dielectric constant. It is clear that by increase $\varepsilon_{\mathrm{r}}$ from 2 (e.g., Teflon) to 30 (e.g., BZT), the $t_{\mathrm{MAX}}$ increases from $\sim 110 \mu \mathrm{m}$ to double of the value. Also, the curve with higher $\varepsilon_{\mathrm{r}}$ has a flatter tail. It means that the measurement is not so sensitive to the sample thickness for higher dielectric constant material, and therefore we get a larger working sample thickness window, which is favorable for sample preparation and handling.

As an example for measurement of loss tangent using this technique, Fig. 5 shows results for temperature dependence of a series of BZT samples with different level of $\mathrm{Ni}$ ion dopings (3-12\%), as well as sapphire spacer as

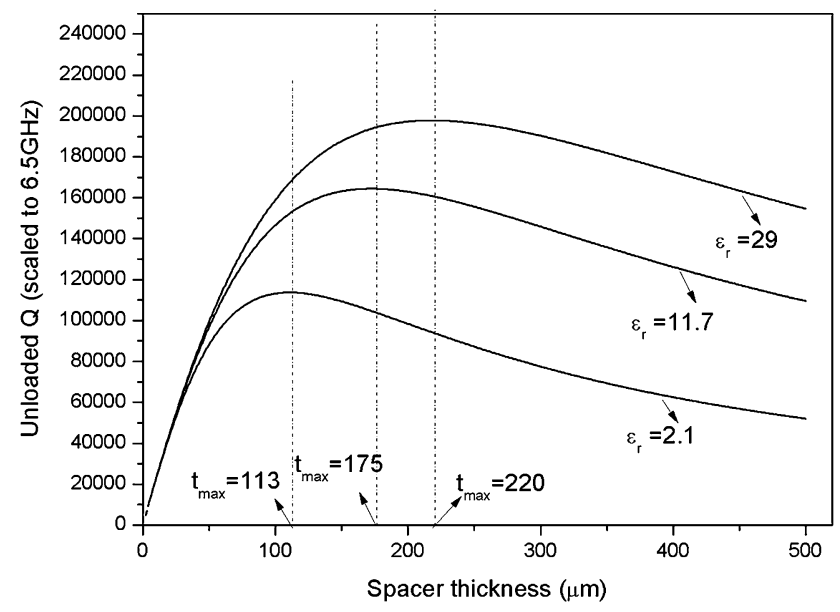

Fig. 4 Unloaded $Q$ of the PPR with different $\varepsilon_{\mathrm{r}}$ of spacers. The curves were drawn based on Eq. (1) with the $R_{\mathrm{s}}$ in this work and $\tan \delta$ of $1.19 \times 10^{-6}$ for the silicon spacer 


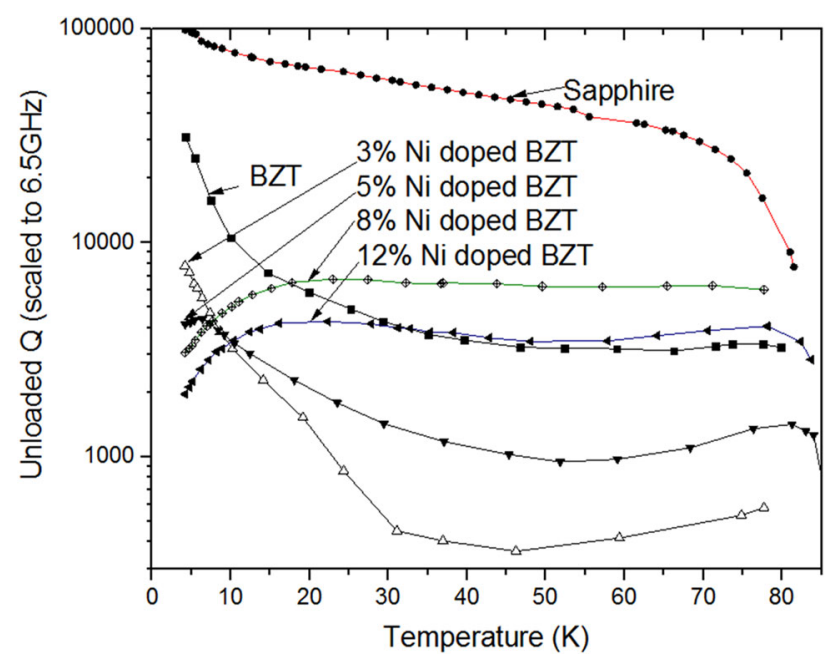

Fig. 5 Unloaded $Q$ of the PPR with different $\varepsilon_{\mathrm{r}}$ of spacers. The curves were drawn based on Eq. (1) with the $R_{\mathrm{s}}$ in this work and $\tan \delta$ of $1.19 \times 10^{-6}$ for the silicon spacer

baseline reference. Although $\mathrm{Nb}$ films achieved the highest $Q$ in our measurement due to super-low surface resistance, we did not choose it for this measurement. Instead, we selected $\mathrm{YBa}_{2} \mathrm{Cu}_{3} \mathrm{O}_{7-\delta}$ (YBCO) superconductor films deposited on sapphire substrates, because YBCO has higher critical temperature ( $93 \mathrm{~K}$ ) compared to $\mathrm{Nb}$ films $(9.3 \mathrm{~K})$, so can easily measure a larger temperature range up to $80 \mathrm{~K}$. As shown by the sapphire spacer baseline reference data (red dot line), the measurement should have enough sensitivity and precision up to $70 \mathrm{~K}$. The most interesting phenomenon one can observe is that, at $4.2 \mathrm{~K}$, with $\mathrm{Ni}$ doping increases from $0 \%$ for undoped BZT to $12 \% \mathrm{Ni}$-doped BZT, the $Q$ factor monotonically decreases and loss tangent goes up. The mechanisms for this phenomenon were well studied in [11-14] which claimed that the reason is due to resonant spin excitations of unpaired transition-metal d electrons by microwave energy in isolated atoms (light doping) or exchange coupled clusters (moderate to high doping). The reason of high loss of undoped and $3 \% \mathrm{Ni}$-doped BZT at 30-70 K temperature range is not clear so far, but probably due to some extrinsic defect-induced absorption mechanisms in these sintered samples, such as polarons, or shallow level point defects.

\section{Conclusion}

Unloaded $Q$ factors of the superconducting PPR have been measured using different dielectric spacer materials with a series of spacer thickness. Among these materials, with ultra-thin Silicon wafers, we obtained a maximum unloaded $Q$ factor of 165,000 (scaled to $6.5 \mathrm{GHz}$ ) when the Silicon spacer is $175 \mu \mathrm{m}$ thick. Parameters of film surface resistance, radiation loss coefficient and dielectric loss tangent were extracted based on the experimental data and theoretical model of unloaded $Q$. The effects of spacer thickness, dielectric constant on the unloaded $Q$ of the PPR have been analyzed in detail. As a material loss tangent measurement technique, it uses smaller sample size $(\sim 1 /$ $500)$ of traditional metal cavity dielectric resonator method, with high sensitivity of $\sim 10^{-5}$ around $7 \mathrm{GHz}$. It can be feasibly loaded into most common cryogenic measurement experiment setup.

Open Access This article is distributed under the terms of the Creative Commons Attribution 4.0 International License (http://crea tivecommons.org/licenses/by/4.0/), which permits unrestricted use, distribution, and reproduction in any medium, provided you give appropriate credit to the original author(s) and the source, provide a link to the Creative Commons license, and indicate if changes were made.

\section{References}

1. Reaney, I.M.: Microwave dielectric ceramics for resonator and filters in mobile phone networks. J. Am. Ceram. Soc. 89, 2063-2072 (2006)

2. Wakino, K., Minai, K., Tamura, H.: Microwave characteristics of ( $\mathrm{Zr}, \mathrm{Sn}) \mathrm{TiO}_{4}$ and $\mathrm{BaO}-\mathrm{PbO}-\mathrm{Nd}_{2} \mathrm{O}_{3}-\mathrm{TiO}_{2}$ dielectric resonators. J. Am. Ceram. Soc. 67, 4 (1984)

3. Liu, L.T., Kopas, C., Singh, R.K., Hanley, R.M., Newman, N.: Growth and characterization of $\mathrm{Ba}\left(\mathrm{Cd}_{1 / 3} \mathrm{Ta}_{2 / 3}\right) \mathrm{O}_{3}$ thin films. Thin Solid Films 520(19), 6153-6157 (2012)

4. Qiu, Min: Analysis of guided modes in photonic crystal fibers using the finite-difference time-domain method. Microw. Opt. Technol. Lett. 30, 5 (2001)

5. Liu, L.T., Wang, M.S., Han, X.Y., Li, J.M.: Recombination of $\mathrm{Br}$-comparison of rate coefficients deduced form the average atom and detailed configuration models. Acta Phys. Sin. 55(5), 2322-2327 (2006)

6. Wang, M.S., Liu, L.T., Li, J.M.: Simulation of ionic populations in hot dense plasmas via a new method beyond the average atom model. Chin. Phys. Lett. 22, 1576-1579 (2005)

7. Tamura, H.: Low temperature properties of microwave dielectrics. Jpn. J. Appl. Phys. 28(Suppl. 2), 21-23 (1989)

8. Wang, X.L., Liu, L.T., Gao, X., Shen, C., Li, J.M.: Interesting features of $n^{2} \mathrm{D}$ Rydberg series fine-structure splittings along the sodium-like isoelectronic sequence. Chin. Phys. Lett. 25(12), 4244-4247 (2008)

9. Wang, M.S., Liu, L.T., Han, X.Y., Li, J.M.: Simulation of hot dense plasma's ionic population: beyond the average atom model. Acta Phys. Sin. 55, 2371-2380 (2006)

10. Manoj Raama Varma: Effect of dopants on the low temperature microwave dielectric properties of $\mathrm{Ba}\left(\mathrm{Zn}_{1 / 3} \mathrm{Ta}_{2 / 3}\right) \mathrm{O}_{3}$ ceramics. J. Mater. Sci. Mater. Electron. 18, 441-446 (2007)

11. Liu, Lingtao, Flores, Marco, Newman, Nathan: Microwave loss in the high-performance dielectric $\mathrm{Ba}\left(\mathrm{Zn}_{1 / 3} \mathrm{Ta}_{2 / 3}\right) \mathrm{O}_{3}$ at $4.2 \mathrm{~K}$. Phys. Rev. Lett. 109, 257601 (2012)

12. Newman, N., Liu, L., Hanley, R., Garg, C.: Resonator techniques to characterize material and device properties at microwave frequencies in the quantum design PPMS measurement system. Appl. Note 40, 1084-1750 (2013)

13. Liu, Lingtao, Matusevich, Alena, Garg, Chirag, Newman, Nathan: The dominance of paramagnetic loss in microwave 
dielectric ceramics at cryogenic temperatures. Appl. Phys. Lett. 101, 252901 (2012)

14. Liu, L., Matusevich, A., Garg, C., Newman, N.: Erratum: The dominance of paramagnetic loss in microwave dielectric ceramics at cryogenic temperatures. Appl. Phys. Lett. 102, 049901 (2013)

15. Taber, R.C.: A parallel plate resonator technique for microwave loss measurement on superconductors. Rev. Sci. Instrum. 61, 2200-2206 (1990)

16. Gao, F., Feng, M., et al.: Mode coupling in superconducting parallel plate resonator in a cavity with outer conductive enclosure. IEEE Trans. Microw. Theory Tech. 44, 6 (1996)

17. Mazierska, J.: Dielectric resonator as a possible standard for characterization of high temperature superconducting films for microwave applications. J. Supercond. 10, 2 (1997)

18. Moeckly, B.H., Oates, D.E., et al.: Microwave properties of $\mathrm{MgB}_{2}$ thin films grown by reactive evaporation. IEEE Trans. Appl. Supercond. 15, 2 (2005)
19. Hernandez, A., Martin, E., Margineda, J., Zamarro, J.M.: Resonant cavities for measuring the surface resistance of metals at X-band frequencies. J. Phys. E Sci. Instrum. 19, 13 (1986)

20. Gao, F., Zhou, Z., Feng, M., Scharen, M., Cardona, A., Forse, R.: Hysteresis effect in microwave power transmission of high temperature superconducting coplanar transmission lines. Appl. Phys. Lett. 67, 2229 (1995)

21. Abbas, F.: Radiation Q-factor of high $\mathrm{T}_{\mathrm{c}}$ superconducting parallel plate resonators. Electron. Lett. 29, 1 (1993)

22. Liu, L.: Mechanisms of Microwave Loss Tangent in High Performance Dielectric Materials. Ph.D. thesis, Arizona State University, Tempe, AZ, USA (2012) 